\title{
Investigação fototérmica de argila vermelha: medidas de propriedades térmicas e estruturais
}

\author{
(Photothermal survey of red clay: measurements of \\ thermal and structural properties)
}

\author{
L. Mota, R. Toledo, R. P. Bastos Filho, H. Vargas, R. T. Faria Jr. \\ Laboratório de Ciências Físicas - LCFIS, Centro de Ciência e Tecnologia - CCT, Universidade Estadual do \\ Norte Fluminense Darcy Ribeiro - UENF, Av. Alberto Lamego 2000, Campos dos Goytacazes, RJ 28013-602 \\ rfaria@uenf.br
}

\begin{abstract}
Resumo
A técnica da célula fotoacústica aberta é uma metodologia fototérmica conhecida, baseada no monitoramento do aumento e da diminuição da temperatura das amostras foram realizadas para obter as propriedades termofísicas, difusividade térmica $\alpha$ e capacidade térmica específica $\rho C$, respectivamente, para caracterizar cerâmicas vermelhas em função da temperatura de queima. Posteriormente, através de uma relação matemática simples, a condutividade térmica $\kappa$ foi avaliada. Valores compreendidos entre $0,0013 \mathrm{~cm}^{2} / \mathrm{s}$ a $0,0036 \mathrm{~cm}^{2} / \mathrm{s}$ e 1,05 a $1,51 \mathrm{~J} / \mathrm{cm}^{3} \mathrm{~K}$ foram obtidos para a difusividade térmica e capacidade térmica específica, respectivamente. Fases cristalinas foram identificadas por difração de raios X, mostrando que a caulinita é a fase predominante. Além disso, a relação entre propriedades térmicas e difratograma foi apresentada e comprovada.

Palavras-chave: propriedades térmicas, open fotoacoustic cell, argila vermelha, difração de raios X.
\end{abstract}

\begin{abstract}
The open photoacoustic cell (OPC) technique and another well known photothermal set up based on the measurement of the increase and decrease temperature of heated samples were carried out to obtain the thermophysical characteristics, thermal diffusivity $\alpha$ and specific heat capacity $\rho C$, respectively, are arranged in order to characterize red ceramics as a function of firing temperature. Thereafter, by means of a simple mathematical relation, we could evaluate the thermal conductivity $\kappa$. Values ranging from $0.0013 \mathrm{~cm}^{2} / \mathrm{s}$ to $0.0036 \mathrm{~cm}^{2} / \mathrm{s}$ and 1.05 to $1.51 \mathrm{~J} / \mathrm{cm}^{3} \mathrm{~K}$ were obtained for thermal diffusivity and specific heat capacity, respectively. Crystalline phases were identified by X-ray diffraction, showing that kaolinite was the predominant phase. Furthermore, the relationship between thermal properties and diffractogram was presented and verified.
\end{abstract}

Keywords: thermal properties, $O P C$, red ceramics, $X R D$.

\section{INTRODUÇÃO}

Observa-se um crescente interesse dos pesquisadores no tocante ao desenvolvimento de novos materiais cerâmicos para aplicações em diversas áreas do conhecimento científico e tecnológico [1-5]. Pode-se notar que já ocorre um direcionamento para o estudo de propriedades térmicas desses novos materiais (difusividade, capacidade específica, condutividade), bem como pesquisas que envolvam a estrutura e mudanças de fase dos mesmos, quando são submetidos a algum tipo de tratamento. Essas pesquisas vêm se destacando como fatores de qualidade, uma vez que cumprem um papel importante à durabilidade, por exemplo, determinando, assim, o tempo de vida útil do produto final $[6,7]$.

O município de Campos dos Goytacazes constitui o maior e um dos mais importantes pólos cerâmicos do estado do Rio de Janeiro. Argilas sedimentares constituem um elevado potencial econômico no município, onde este tipo de solo é abundante e usado em aplicações industriais como produção de cerâmica, principalmente tijolos e telhas [8]. Geograficamente, o município de Campos fica localizado em uma região de clima tropical. Como consequiência, as construções civis estão freqüentemente submetidas a uma constante radiação solar e, portanto, propriedades térmicas do material, empregado para este fim, exercem função importante com relação ao clima interno das residências.

A argila é submetida a elevadas temperaturas para atingir a qualidade desejável $[9,10]$. Com relação ao processo de produção, foram investigadas as propriedades térmicas e transições de fase que ocorreram na estrutura do material, cuja aplicação final destina-se à indústria de construção civil. É necessário avaliar estes parâmetros uma vez que, além de serem propriedades singulares para cada material, são extremamente sensíveis a variáveis composicionais e microestruturais, bem como às condições de processamento [11].

Neste trabalho, uma configuração baseada no processo de transmissão de calor, denominada célula fotoacústica 
aberta (OPC), foi empregada para a geração e detecção de ondas térmicas [12]. Esta metodologia tem sido amplamente utilizada nos últimos anos como uma poderosa ferramenta no estudo e determinação de propriedades térmicas para uma ampla variedade de materiais [13-16]. Ela consiste em utilizar a câmara de ar de um microfone de eletreto comercial como meio transdutor de uma célula fotoacústica convencional. O princípio básico desta técnica reside na conversão de radiação periodicamente modulada em energia térmica, através de processos de relaxação nãoradiativos [12].

\section{MATERIAL E MÉTODOS}

\section{Matéria-prima}

A matéria-prima utilizada para a confecção dos corpos de prova consiste de uma argila vermelha, coletada de uma camada situada entre 1,2 m e 2,7 m abaixo da superfície de uma jazida localizada no município de Campos dos Goytacazes, na região Norte Fluminense. O material em sua forma natural foi levado a uma estufa por $24 \mathrm{~h}$ sob $110{ }^{\circ} \mathrm{C}$ com o propósito de eliminar a umidade higroscópica (água adsorvida). Após este tratamento, o material foi destorroado com pilão manual e peneirado através de uma peneira de abertura nominal $75 \mu \mathrm{m}$ (mesh $200 \mathrm{ABNT}$ ). Este procedimento foi realizado com o objetivo de suprimir a presença de areia e outros particulados com diâmetros maiores, favorecendo a presença da fração argila e proporcionando uma maior homogeneidade no tamanho dos grãos.

\section{Preparação das amostras}

As amostras, que consistem de discos (pastilhas) com $10 \mathrm{~mm}$ de diâmetro e espessuras compreendidas entre $270 \mu \mathrm{m}$ e $420 \mu \mathrm{m}$ aproximadamente, foram preparadas com o auxílio de uma prensa (Shimadzu). Elas tiveram suas massas medidas, padronizadas em torno de $90 \mathrm{mg}$ e submetidas a uma pressão de 9 ton por 10 min. Este procedimento foi feito para garantir uniformidade nas dimensões das amostras. Após isto, as pastilhas passaram por um tratamento térmico em temperaturas variando entre $300{ }^{\circ} \mathrm{C}$ e $1100{ }^{\circ} \mathrm{C}$.

\section{Medidas de difusividade térmica}

$\mathrm{O}$ arranjo experimental utilizado para as medidas de difusividade térmica é ilustrado na Fig. 1. A fonte de excitação adotada foi um laser de He-Ne (Unilaser 0.25), dotado de uma linha de emissão em torno de 632,8 nm. Antes de atingir a superfície da amostra, a radiação passa por um modulador mecânico "chopper" (EG \& G Instr. 651), responsável por modular a radiação incidente.

Como resultado do aquecimento da amostra pela absorção da luz modulada, a pressão dentro da célula oscila com a mesma frequiência de oscilação do modulador mecânico e assim pode ser detectada pelo microfone de eletreto. $\mathrm{O}$ sinal

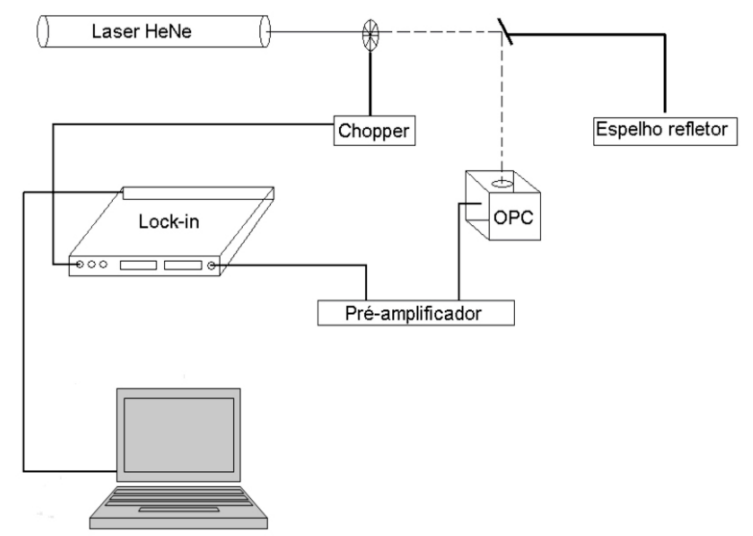

Figura 1: Arranjo experimental para as medidas de difusividade térmica.

[Figure 1: Experimental setup for thermal diffusivity measurements.]

fotoacústico resultante deste processo é alimentado por um pré-amplificador (FET) e em seguida conduzido diretamente para um amplificador lock-in (Perkin Elmer Instr. 5210), onde é possível obter tanto a fase quanto a amplitude do sinal fotoacústico [17], cujos valores foram armazenados em um microcomputador.

De acordo com o modelo, considerando a difusão térmica como mecanismo predominante para a geração do sinal fotoacústico [18], a equação que governa a flutuação de pressão na câmara de ar é [17, 19, 20]:

$$
\delta \mathrm{P}=\frac{\gamma \mathrm{P}_{0} \mathrm{I}_{0}\left(\alpha_{\mathrm{g}} \alpha_{\mathrm{s}}\right)^{\frac{1}{2}}}{2 \pi l_{\mathrm{g}} \mathrm{T}_{0} \mathrm{k}_{\mathrm{s}} f} \frac{\exp \mathrm{j}(\omega \mathrm{t}-\pi / 2)}{\operatorname{senh}\left(\sigma_{\mathrm{s}} l\right)}
$$

onde $\gamma$ é a razão entre os calores específicos, $P_{0}$ a pressão ambiente, $T_{0}$ a temperatura ambiente, $I_{0}$ a intensidade de luz absorvida, $f$ a freqüência de modulação, e $l_{i}, k_{i}, \alpha_{i}$ são o comprimento, a condutividade térmica e a difusividade térmica do meio $i$, respectivamente. Aqui, o subscrito $i$ representa a $\operatorname{amostra}(s)$ e o gás $(g)$, e $\sigma_{i}=(1+j) a_{i}, a_{i}=\left(\pi f / \alpha_{i}\right)^{1 / 2}$ designa o coeficiente complexo de difusão térmica do meio $i$. Assume-se na equação A que as amostras sejam opticamente opacas $\left(l_{\beta}<<\right.$ $\left.l_{s}\right)$. Para amostras termicamente finas $\left(\mu_{s}>l_{s} ; f<f_{c}\right)$, a equação Á é escrita como $[17,19,20]$ :

$$
\delta \mathrm{P}=\frac{\gamma \mathrm{P}_{0} \mathrm{I}_{0}\left(\alpha_{\mathrm{g}}\right)^{\frac{1}{2}} \alpha_{\mathrm{s}}}{2 \pi^{\frac{2}{3}} l_{\mathrm{g}} l_{\mathrm{s}} \mathrm{T}_{0} \mathrm{k}_{\mathrm{s}}} \frac{\exp \mathrm{j}(\omega \mathrm{t}-3 \pi / 4)}{f^{\frac{3}{2}}}
$$

Neste caso, a amplitude do sinal fotoacústico decresce proporcionalmente a $f^{-1,5}$. Por outro lado, em regiões de freqüência acima da frequiência característica $f_{c}$, a amostra é denominada como termicamente grossa e a equação correspondente a esta situação é $[17,19,20]$ :

$$
\delta \mathrm{P}=\frac{\gamma \mathrm{P}_{0} \mathrm{I}_{0}\left(\alpha_{\mathrm{g}} \alpha_{\mathrm{s}}\right)^{\frac{1}{2}}}{\pi l_{\mathrm{g}} \mathrm{T}_{0} \mathrm{k}_{\mathrm{s}}} \frac{\exp -l_{\mathrm{s}} \sqrt{\pi f / \alpha_{\mathrm{s}}}}{f} \exp \mathrm{j}\left(\omega \mathrm{t}-\frac{\pi}{2}-1_{\mathrm{s}} \alpha_{\mathrm{s}}\right)(\mathrm{C})
$$


A amplitude do sinal fotoacústico para uma amostra termicamente grossa decresce exponencialmente com a freqüência de modulação, ou seja, $S_{P A} \propto(1 / f) \exp (-b \sqrt{ } f)$, sendo $b=l_{s} \sqrt{ } \pi / \alpha_{s}$. Neste caso, $a_{s}$ é obtido a partir do ajuste dos resultados experimentais do coeficiente (b) no argumento da exponencial $(-b \sqrt{ } f)$. Com o objetivo de certificar e garantir o regime termicamente grosso, valores de difusividade térmica foram utilizados [21]. A frequiência de corte encontrada para a cerâmica vermelha foi em torno de $5,87 \mathrm{~Hz}$, ou seja, como todas as medidas realizadas neste trabalho estãocompreendidas entre 20 e $50 \mathrm{~Hz}$, o regime foi garantido.

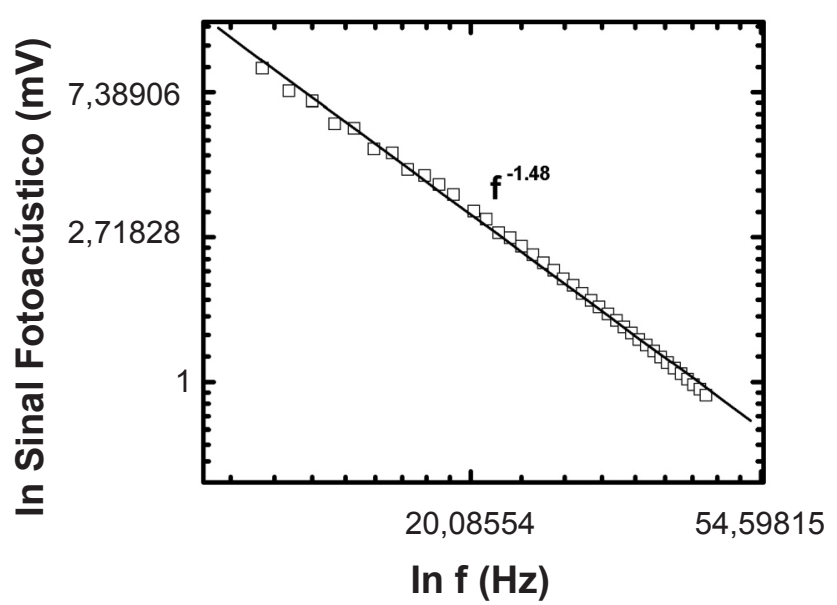

Figura 2: Dependência do sinal fotoacústico com a freqüência de modulação para uma amostra de alumínio com $25 \mu \mathrm{m}$ de espessura.

[Figure 2: Photoacoustic signal dependence with the modulation frequency for a $25 \mathrm{~m}$ thick aluminum sample.]

Quando os valores de difusividade térmica são obtidos a partir da amplitude do sinal fotoacústico, é necessário prestar atenção na resposta de frequiência não linear em relação às vibrações acústicas. Praticamente, todos os microfones apresentam esta irregularidade. Por este motivo, uma medida de calibração foi realizada. A Fig. 2 mostra a dependência do sinal fotoacústico com a freqüência de modulação para uma amostra de alumínio [22, 23].

Para a região de frequiências trabalhadas, o sinal exibiu uma dependência próxima de $f^{-1,5}$. Este é o comportamento típico que se espera de acordo com o modelo de difusão térmica para uma amostra termicamente fina. De fato, para uma amostra de alumínio com $25 \mu \mathrm{m}$ de espessura e uma difusividade térmica [24] de $93,28.10^{-6} \mathrm{~m} / \mathrm{s}^{2}$ a frequiência característica $f_{c}$ para a transição entre os regimes termicamente fino e grosso é aproximadamente 47,5 kHz.

\section{Medidas de capacidade térmica específica}

Na década de 1990, com base na ref. [25], como forma de complementar suas medidas fotoacústicas de propriedades térmicas em sólidos, foi desenvolvida uma metodologia fototérmica de iluminação contínua da amostra em vácuo para medir o calor específico [26]. Esta variante do método

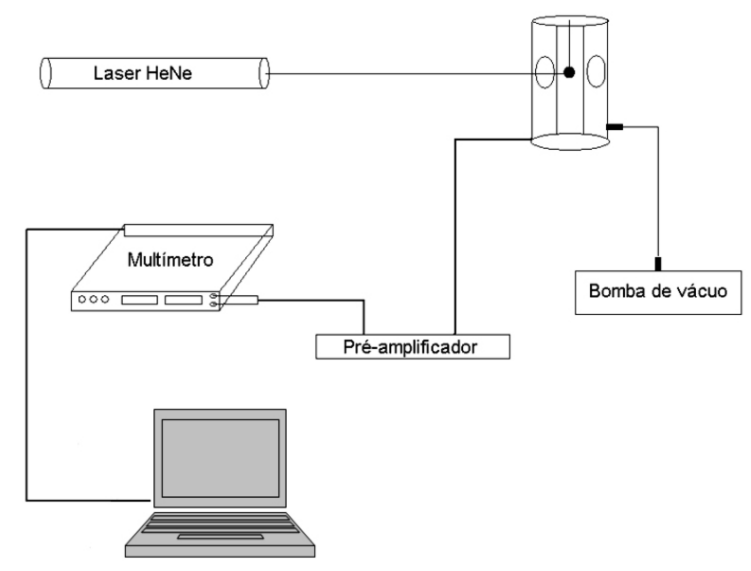

Figura 3: Arranjo experimental para as medidas de capacidade térmica específica.

[Figure 3: Experimental arrangement for specific heat capacity measurements.]

de relaxação da temperatura, primeiramente proposto por Bachman [27], foi usada para a caracterização de diversos materiais tais como semicondutores [28], madeira [29], zeólitas [30], polímeros [31], entre outros. A Fig. 3 apresenta a montagem experimental empregada que nos permite avaliar este parâmetro.

A amostra é pintada com uma fina camada de tinta preta com espessura desprezível, para garantir uma boa absorção superficial, bem como fazer com que a emissividade se aproxime da unidade $\left(\varepsilon_{m}=1\right)$. Em seguida, a amostra é colocada suspensa adiabaticamente dentro de um frasco dewar onde é feito vácuo. Sob tais condições o principal mecanismo de troca de calor é o de radiação. O frasco tem uma entrada óptica que possibilita o aquecimento da amostra por meio de um laser de He-Ne. Um termopar, acoplado no lado oposto ao iluminado, permite o monitoramento da temperatura da amostra ao longo do tempo. Os valores da temperatura em função do tempo são então monitorados e armazenados em um microcomputador. Cuidado deve ser exercido para prevenir que o feixe de luz que aquece a amostra não incida também no termopar. Esta condição é garantida, fazendo a radiação incidir no centro da amostra. Sendo as espessuras das amostras tipicamente da ordem de micrometros, muito menores que seus diâmetros $(10 \mathrm{~mm})$, a equação unidimensional de difusão de calor com perda por radiação pôde ser aplicada nesse estudo [26]. O aumento e a diminuição da temperatura são dados conforme as seguintes expressões:

$$
\Delta \mathrm{T} \uparrow=\frac{\mathrm{I}_{0}}{\mathrm{H}}\left(1-\exp \left[-\frac{\mathrm{t}}{\tau}\right]\right)
$$

$$
\left.\Delta \mathrm{T} \downarrow=\frac{\mathrm{I}_{0}}{\mathrm{H}} \exp \left[-\frac{\mathrm{t}}{\tau}\right]\right)
$$

onde $I_{0}$ é a intensidade da luz incidente e $\tau=l_{s} \rho c /(2 H)$, a 
constante de tempo, $H=4 \sigma T_{0}^{3}$, coeficiente de transferência de calor, sendo $\sigma$ a constante de Stefan-Boltzmann e $T_{0}$, a temperatura ambiente. $O$ parâmetro $\tau$ é determinado ajustando as equações $\mathrm{D}$ ou $\mathrm{E}$ aos resultados experimentais obtidos e compatíveis com o regime de temperatura respectivo.

\section{Medidas de condutividade}

Nenhum método fototérmico pode fornecer a condutividade térmica $k$ de uma amostra diretamente, uma vez que este parâmetro está sempre acoplado com a capacidade térmica. A condutividade térmica depende do calor específico, que é afetado pelas variáveis composicionais e microestruturais do material (impurezas, contorno de grãos), que modificam a velocidade e o livre caminho médio dos portadores [24]. Assim, a partir da determinação da difusividade térmica $\alpha$ e da capacidade térmica específica $\rho c$, pode-se então determinar a condutividade térmica $k$ do material por meio da seguinte equação:

$$
k=\alpha \rho c
$$

ou seja, através de metodologias relativamente de baixo custo, pode-se identificar de forma precisa três importantes propriedades de um determinado material.

\section{Medidas de difração de raios $X$}

A análise estrutural foi obtida com o auxílio de um difratômetro convencional de raios X, com geometria BraggBrentano (Seifert URD 65), com radiação Cuk $_{\alpha}(1,54056 \AA)$, com monocromador de grafite no feixe secundário, operando a $30 \mathrm{kV} / 40 \mathrm{~mA}$. As amostras, que foram preparadas em forma de discos compactados e tratadas termicamente, foram trituradas, pulverizadas, homogeneizadas por peneiramento e depositadas em lâmina de vidro com pequena cavidade circular, para cada temperatura investigada. Todas as medidas foram feitas sob as mesmas condições experimentais, ou seja, ângulo de varredura $3^{\circ} \leq 2 \theta \leq 75^{\circ}$ e passo $0,03^{\circ} / 2 \mathrm{~s}$ em temperatura ambiente.

\section{RESULTADOS E DISCUSSÃO}

A fase cristalina predominante na estrutura do material antes do tratamento térmico foi a caulinita, associada à presença de quartzo, anatásio e gibsita, além de minerais micáceos (mica e/ou ilita). Picos de caulinita bastante pronunciados são vistos nos difratogramas (Fig. 4) até temperaturas em torno de $400{ }^{\circ} \mathrm{C}$. Considerou-se este argilomineral como sendo o principal responsável pelos valores de difusividade térmica encontrados neste intervalo de temperatura, como resultado de um solo quaternário bem cristalizado [32]. Em trabalho anterior [21] foi mostrado que a argila encontrada no município de Campos, RJ, apresenta elevada cristalinidade neste intervalo de temperatura; sabese ainda que materiais com estruturas cristalinas mais bem

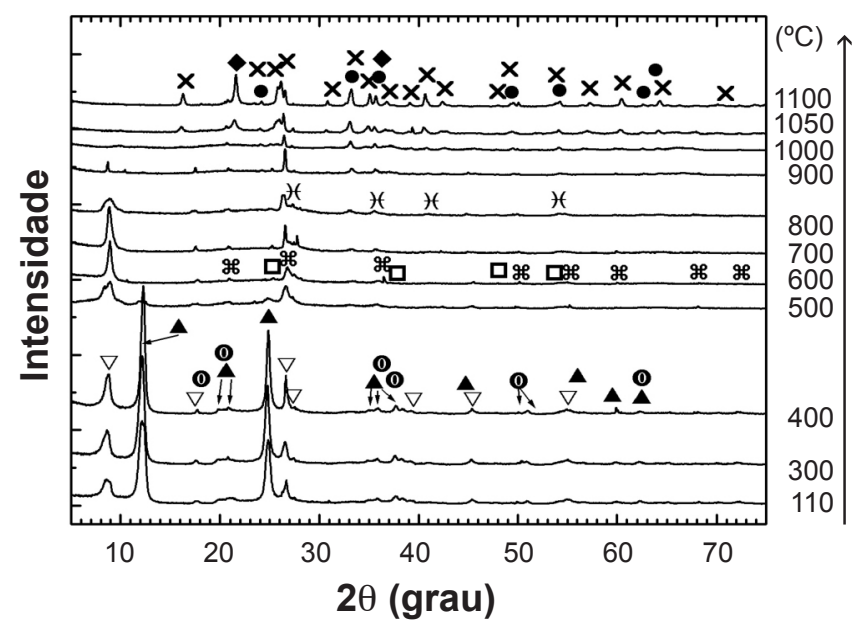

Figura 4: Difratogramas de raios $\mathrm{X}$ da amostra natural $\left(110^{\circ} \mathrm{C}\right) \mathrm{e}$ das amostras queimadas em diferentes temperaturas.

[Figure 4: X-ray diffraction patterns of natural $\left(110^{\circ} \mathrm{C}\right)$ and for varied firing temperatures samples.]

organizadas favorecem o transporte de energia térmica através de seu arcabouço [33], corroborando os resultados de difusividade térmica.

Entre $450{ }^{\circ} \mathrm{C}$ e $550{ }^{\circ} \mathrm{C}$ foi observada a desidroxilação da caulinita, originando a formação de uma fase amorfa denominada metacaulinita devido à perda de hidroxilas, em forma de vapor de água $[34,35]$. Os radicais hidroxila, que estão presentes na estrutura da caulinita coordenados em torno de seus íons alumínio, transformam-se em água e são expelidos do cristal com elevada absorção de energia e perda de massa [36]. A reação da equação $G$ mostra a formação da metacaulinita a partir da caulinita, cabendo ressaltar que não se trata de uma reação de equilíbrio, não sendo portanto, reversível.

$$
2 \mathrm{Al}_{2}(\mathrm{OH})_{4} \mathrm{Si}_{2} \mathrm{O}_{5} \stackrel{500^{\circ} \mathrm{C}}{\longrightarrow} 2 \mathrm{Al}_{2} \mathrm{Si}_{2} \mathrm{O}_{7}+4 \mathrm{H}_{2} \mathrm{O}
$$

Acredita-se ser essa fase meta-estável a responsável pelo menor valor alcançado para a difusividade térmica (Fig. 5), e mesmo porque já é conhecido que as fases amorfas apresentam menores difusividades do que em fases com caráter mais cristalino [33].

Entre $600{ }^{\circ} \mathrm{C}$ e $700{ }^{\circ} \mathrm{C}$ o difratograma revelou picos bem definidos de quartzo e mica e alguns traços de anatásio. Vê-se ainda que a forte presença de caulinita já não se fez evidente. Ocorre também que nestas temperaturas o material já sofreu um processo de redução de impurezas, caracterizado pela combustão de matéria orgânica entre $300{ }^{\circ} \mathrm{C}$ e $500{ }^{\circ} \mathrm{C}$ [37]. Com relação ao comportamento da difusividade térmica, observou-se um pequeno aumento em seus valores nestas temperaturas. Sugere-se assim, que este fato está associado ao menor grau de impurezas contidas no material cerâmico, favorecendo a uma pequena melhora na distribuição dos poros. Como exemplo, apresenta-se na Fig. 6 o ajuste dos resultados experimentais (símbolos abertos) de acordo com a equação 3 (linha sólida) para as amostras tratadas termicamente em $600{ }^{\circ} \mathrm{C}, 700{ }^{\circ} \mathrm{C}$ e $800{ }^{\circ} \mathrm{C}$. 


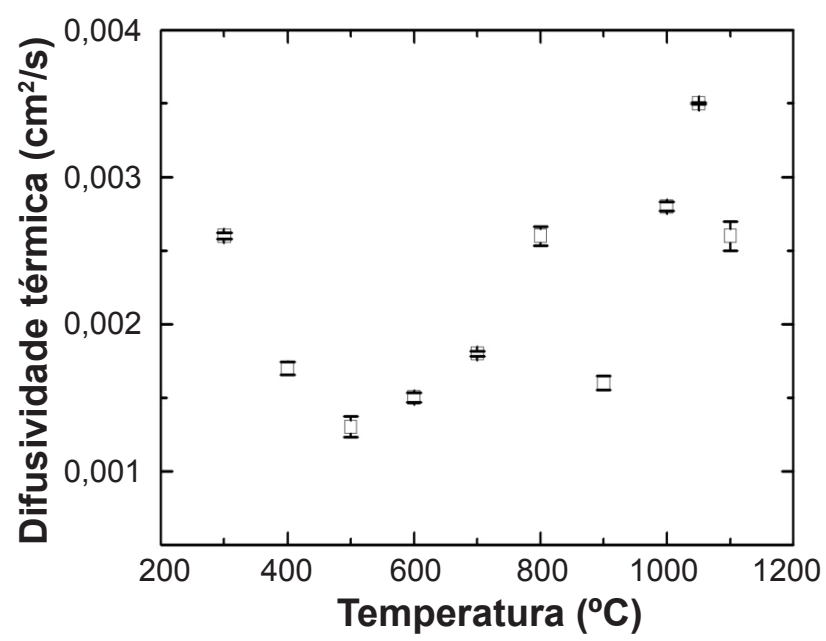

Figura 5: Valores da difusividade térmica em função da temperatura de queima.

[Figure 5: Thermal diffusivity values as a function of firing temperature.]

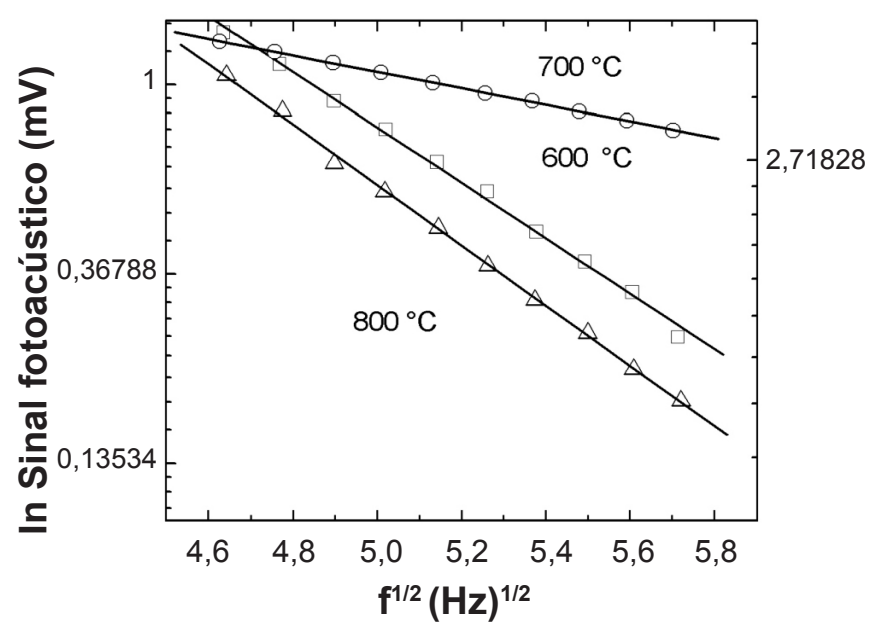

Figura 6: Ajuste dos resultados experimentais (símbolos abertos) de acordo com a equação 3 (linha sólida) para as amostras de $600{ }^{\circ} \mathrm{C}, 700{ }^{\circ} \mathrm{C}$ e $800{ }^{\circ} \mathrm{C}$.

[Figure 6: Fittings of the experimental data (open symbols) following equation 3 (solid line) for the $600{ }^{\circ} \mathrm{C}, 700^{\circ} \mathrm{C}$ and $800{ }^{\circ} \mathrm{C}$ samples.]

A presença de traços de hematita já em $800{ }^{\circ} \mathrm{C}$ pode estar associada à evolução de alguma quantidade de ilita presente na matéria-prima, além da possibilidade de ser decorrente da presença de goetita na matéria-prima, não detectada por difração de raios $X$, por comportar-se como material de fraca cristalinidade. Não há evidências de que a hematita originese propriamente da ilita, porém surge de um óxido de ferro [38] tal como a goetita ou limonita, que por sua vez, estão diretamente conectados com a ilita. O difratograma revelou ainda uma quantidade de mica remanescente. Essa fase associada com o aumento de temperatura, favorece a um leve aparecimento de fase vítrea [38], porém mantendo até essa temperatura um certo grau de organização estrutural.
Atribui-se a essa fase vítrea combinada ao arranjo estrutural o mérito pelo aumento observado no perfil da difusividade térmica.

Com a quebra de estrutura e perda de cristalinidade devido à fase fundente iniciada a partir de $800^{\circ} \mathrm{C}$, ocorre em $900^{\circ} \mathrm{C}$ uma desorganização estrutural que é acompanhada por uma queda acentuada em $\alpha$, evidenciando que tal propriedade é também sensível a essa transição. Entre $900{ }^{\circ} \mathrm{C}$ e $1000{ }^{\circ} \mathrm{C}$ a difusividade experimentou um aumento significativo. Muitos autores já discutiram a respeito da possível formação de uma fase de transição denominada espinélio [32, 34, 35, $39,40]$, seguida por um processo de rearranjo entre os átomos de silício-alumínio e uma conseqüente recristalização. Também tem-se nessas temperaturas o início de formação de fase líquida, favorecendo o transporte de calor através do material cerâmico. A reação mostrada na equação $\mathrm{H}$ ilustra que a partir da metacaulinta $\left(2 \mathrm{Al}_{2} \mathrm{Si}_{2} \mathrm{O}_{7}+4 \mathrm{H}_{2} \mathrm{O}\right)$ ocorre a formação da fase espinélio $\left(\mathrm{Al}_{4} \mathrm{Si}_{3} \mathrm{O}_{12}+\mathrm{SiO}_{2}\right)$,

$$
2 \mathrm{Al}_{2} \mathrm{Si}_{2} \mathrm{O}_{7}+4 \mathrm{H}_{2} \mathrm{O} \stackrel{\approx 980^{\circ} \mathrm{C}}{\longrightarrow} \mathrm{Al}_{4} \mathrm{Si}_{3} \mathrm{O}_{12}+\mathrm{SiO}_{2}
$$

Entre $1000{ }^{\circ} \mathrm{C}$ e $1100{ }^{\circ} \mathrm{C}$ foi notada a formação de novas fases com o processo cerâmico. Essas fases foram mulita, cristobalita, além da hematita melhor definida, como observadas no difratograma. Ressalta-se que em $1050{ }^{\circ} \mathrm{C}$ o máximo valor para a difusividade térmica foi atingido. Sugere-se que esse resultado está intimamente relacionado com a fase vítrea mais proeminente formada entre $1040^{\circ} \mathrm{C} \mathrm{e}$ $1080{ }^{\circ} \mathrm{C}$, propiciando a sinterização das amostras e coerente com o aumento da cristalinidade vista nos difratogramas (Fig. 4). Em $1100{ }^{\circ} \mathrm{C}$, a difusividade térmica apresentou um decréscimo. Tal comportamento pode ser caracterizado pela reação da equação I:

$$
\mathrm{Al}_{4} \mathrm{Si}_{3} \mathrm{O}_{12} \underset{\approx 1100^{\circ} \mathrm{C}}{\longrightarrow} \frac{2}{3}\left[3 \mathrm{Al}_{2} \mathrm{O}_{3} 2 \mathrm{Si}_{2}\right]+\frac{5}{3} \mathrm{SiO}_{2}
$$

Nessa temperatura, a formação da mulita $2 / 3\left(3 \mathrm{Al}_{2} \mathrm{O}_{3} 2 \mathrm{Si}_{2}\right)$ e cristobalita $\left[5 / 3 \mathrm{SiO}_{2}\right]$ têm início a partir da fase espinélio $\left(\mathrm{Al}_{4} \mathrm{Si}_{3} \mathrm{O}_{12}\right)$ como reportado em trabalho anterior [41]. A principal hipótese é que houve uma redução de fase líquida e também dos poros, e o conseqüente crescimento de grãos, caracterizados pelo surgimento das fases cristalinas mais bem definidas mulita, cristobalita e hematita, além do quartzo remanescente.

A capacidade térmica específica $\rho c$ ilustra o quanto uma amostra de um determinado material consegue armazenar de energia térmica, sem sofrer alteração em sua temperatura. Os resultados obtidos para esta propriedade estão mostrados na Fig. 7. Vale ressaltar que os valores estão oscilando em torno da unidade. De fato, em se tratando de uma mesma matériaprima, essa propriedade apresenta valores bastante próximos, independente se a estrutura está cristalina ou amorfa [42]. Com isto, pode-se inferir que, sendo a outra característica térmica, ou seja, a condutividade obtida indiretamente a partir da equação $\mathrm{F}$, o seu perfil será semelhante ao perfil de $\alpha$. A Fig. 8 ilustra o ajuste dos resultados experimentais (símbolos abertos) de acordo com as equações D e E (linhas 


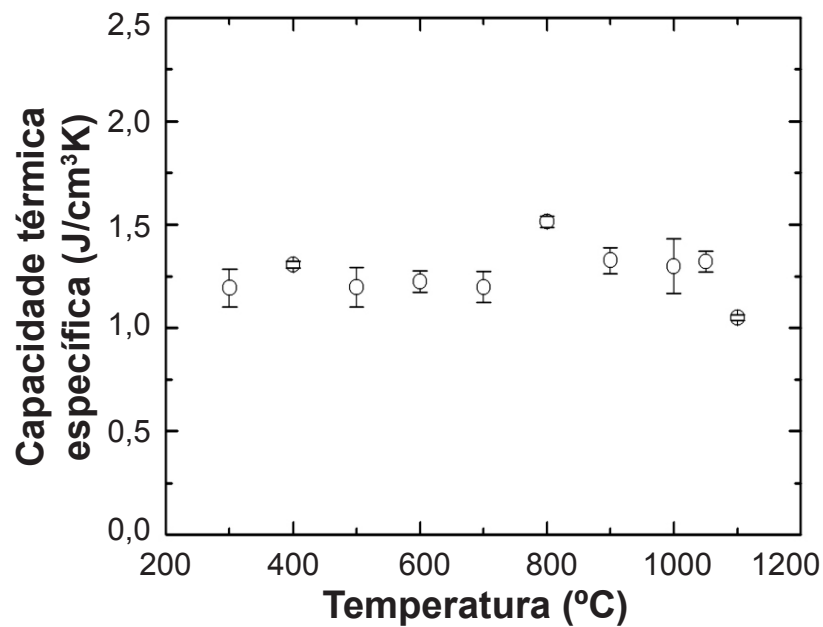

Figura 7: Valores da capacidade térmica específica em função da temperatura de queima.

[Figure 7: Specific heat capacity values as a function of firing temperature.]

sólidas) para a amostra queimada em $400{ }^{\circ} \mathrm{C}$.

A Fig. 9 mostra a evolução do comportamento de $k$. Talvez seja esta a característica de maior interesse prático e a mais difundida. Esta propriedade está associada com o fenômeno de tranporte de calor [43] a partir de regiões de alta temperatura para regiões de baixa temperatura em um determinado meio. Normalmente, este transporte em cerâmica vermelha é devido aos fônons (vibração da rede cristalina), pelo fato deste material carecer de elétrons livres. Assim sendo, são esperados valores, comparativamente muito baixos, de condutividade térmica para a cerâmica vermelha (em torno de $10^{-3} \mathrm{~W} / \mathrm{cmK}$ ) [44].

Mesmo sendo uma medida indireta, os resultados obtidos mostram bastante concordância com aqueles publicados

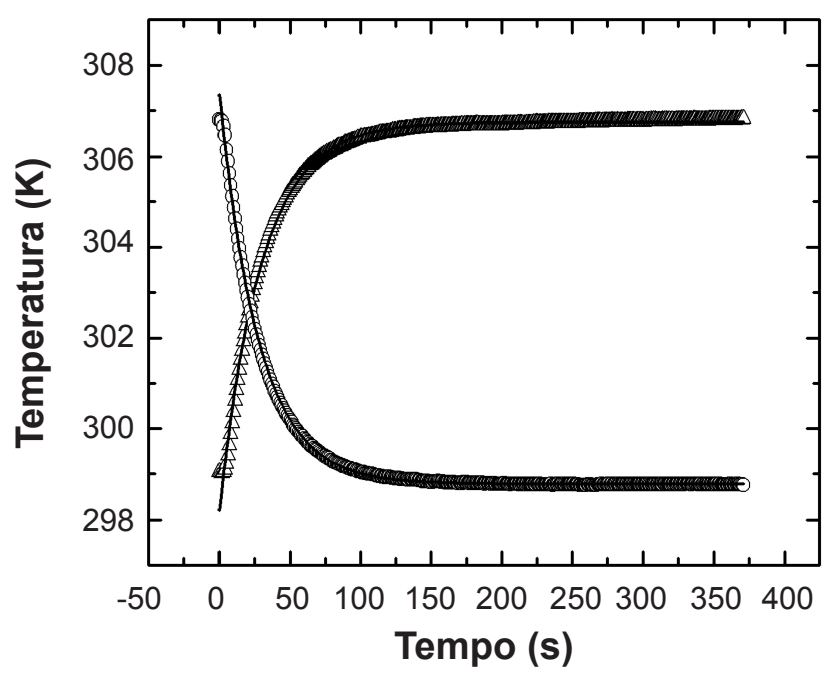

Figura 8: Dados experimentais e linhas teóricas das temperaturas de subida (triângulos) e descida (círculos) em função do tempo para a amostra queimada em $1100{ }^{\circ} \mathrm{C}$.

[Figure 8: Experimental data and theoretical lines of rising (triangles) and decreasing (circles) temperatures as a function of time for the $1100{ }^{\circ} \mathrm{C}$ sample.]

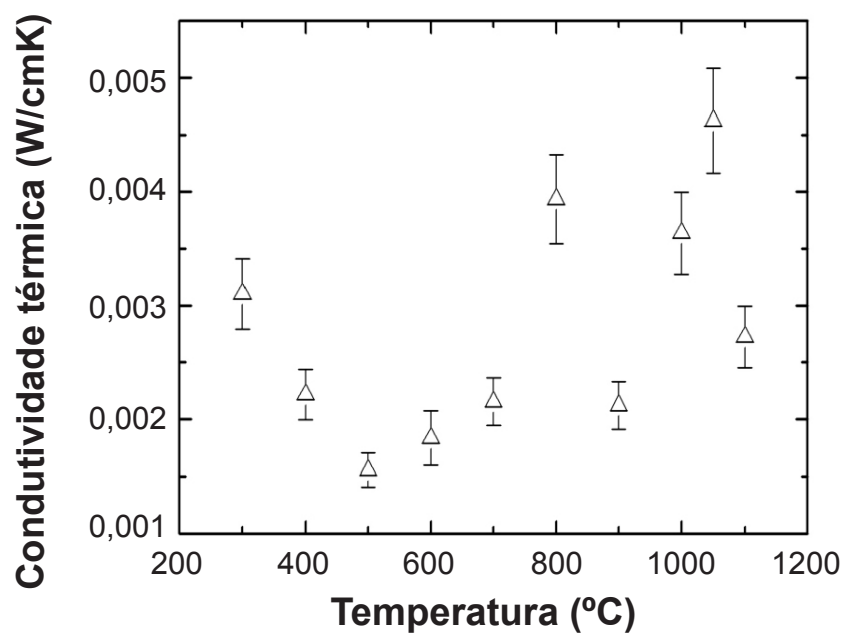

Figura 9: Valores da condutividade térmica em função da temperatura de queima.

[Figure 9: Thermal conductivity values as a function of firing temperature.]

anteriormente [44]. Isso implica que os resultados alcançados para a difusividade térmica e capacidade térmica específica mostraram confiabilidade e que a célula fotoacústica aberta (OPC) é uma metodologia adequada também para o estudo de materiais cerâmicos.

\section{CONCLUSÕES}

Foram investigadas a evolução do comportamento de algumas propriedas térmicas (principalmente a difusividade térmica) e também mudanças estruturais que ocorrem no material cerâmico durante o processo de queima, podendo contribuir com alguns aspectos considerados relevantes para se produzir um produto com relativa qualidade. Foi identificado que o principal argilomineral presente na matéria-prima foi a caulinita, que com uma estrutura cristalina organizada (solo quaternário) favorece a difusão de calor através de seu interior. Em seguida, o material cerâmico experimentou uma quebra estrutural devido à desidroxilação da caulinta e formação de uma fase amorfa. Podemos ver que esta transição foi $\operatorname{logo}$ percebida por $\alpha$, mostrando sua sensibilidade em detectar mudanças de fase. Entre $900{ }^{\circ} \mathrm{C}$ e $1000{ }^{\circ} \mathrm{C}$ notou-se que a difusividade sofreu um aumento. Este evento está associado à formação de uma fase espinélio, seguida por um processo de recristalização, além do início de formação de fase líquida, favorecendo o transporte de calor através do material cerâmico. Foi ainda observado que em $1050{ }^{\circ} \mathrm{C}$ o máximo valor para a difusividade térmica foi atingido. Sugere-se que este resultado está intimamente relacionado com a fase vítrea mais proeminente formada entre $1040{ }^{\circ} \mathrm{C}$ e $1080{ }^{\circ} \mathrm{C}$, tornando possível a sinterização das amostras, coerente com o aumento da cristalinidade vista no difratograma. Em $1100{ }^{\circ} \mathrm{C}$ a difusividade térmica experimentou um acentuado decréscimo. Sugere-se que houve uma redução de fase líquida e também dos poros e o conseqüente aparecimento 
dos grãos cerâmicos caracterizados pelo surgimento das fases cristalinas mais bem definidas mulita, cristobalita e hematita, além do quartzo remanescente. Por fim sugere-se que temperaturas adequadas para se produzir um material cerâmico com relativa qualidade sejam superiores a $900{ }^{\circ} \mathrm{C}$, porém evitando temperaturas em torno de $1050{ }^{\circ} \mathrm{C}$.

\section{AGRADECIMENTOS}

À FAPERJ, ao CNPq, e à Universidade Estadual do Norte Fluminense Darcy Ribeiro (UENF).

\section{REFERÊNCIAS}

[1] N. A. George, T. Paul, P. Radhakrishnan, V. P. N. Nampoori, C. P. G. Vallabhan, J. Mater. Sci. Lett. 19 (2000) 499.

[2] E. García, M. I. Osendi, P. Miranzo, J. Appl. Phys. 92 (2002) 2346.

[3] P. G. Bison, F. Cernuschi, E. Grinzato, S. Marinetti, D. Robba, Infrared Phys. Techn. 49 (2007) 286.

[4] B. Li, A. Mandelis, Z. Z. Kish, J. Appl. Phys. 95 (2004) 1042.

[5] R. T. Faria Jr., M. F. Rodrigues, I. A. Esquef, H. Vargas, M. Filgueira, Int. J. Refractory Metals Hard Mater. 23 (2005) 115.

[6] M. Akiyoshi, I. Takagi, T. Yano, N. Akasaka, Y. Tachi, Fusion Eng. Design 81 (2006) 321.

[7] C. Pradère, J. M. Goyhénèche, J. C. Batsale, S. Dilhaire, R. Pailler, Int. J. Thermal Sci. 45 (2006) 443.

[8] R. Toledo, D. R. dos Santos, R. T. Faria Jr., J. G. Carrió, L. T. Auler, H. Vargas, Appl. Clay Sci. 27 (2004) 151.

[9] S. N. Monteiro, C. M. F. Vieira, Appl. Clay Sci. 27 (2004) 229.

[10] C. M. F. Vieira, T. M. Soares, R. Sánchez, S. N. Monteiro, Mater. Sci. Eng. A 373 (2004) 115.

[11] V. Anjos, M. J. V. Bell, E. A. de Vasconcelos, E. F. da Silva Jr., A. A. Andrade, R. W. A. Franco, M. P. P. Castro, I. A. Esquef, R. T. Faria Jr., Microelectronics J. 36 (2005) 977. [12] H. Vargas, L. C. M. Miranda, Phys. Reports 161 (1988) 43.

[13] N. A. George, Smart Mater. Structures 11 (2002) 561.

[14] V. Dossetti-Romero, J.A. Méndez-Bermúdez, E. LópezCruz, J. Phys.: Condensed Matter 14 (2002) 9725.

[15] S. D. George, S. Saravanan, M. R. Anantharaman, S. Venkatachalam, P. Radhakrishnan, V. P. N. Nampoori, C. P. G. Vallabhan, Phys. Rev. B 69 (2004) 235201-1.

[16] A. Calderón, R. A. Muñoz-Hernández, S. A. Tomás, A. Cruz-Orea, F. S. Sinencio, J. Appl. Phys. 84 (1998) 6327.

[17] M. D. da Silva, I. N. Bandeira, L. C. M. Miranda, J. Phys. E: Sci. Instr. 20 (1987) 1476.

[18] A. Rosencwaig, A. Gersho, J. Appl. Phys. 47 (1976) 64.

[19] M. V. Marquezini, N. Cella, A. M. Mansanares, H. Vargas, L. C. M. Miranda, Measurement Sci. Techn. 2 (1991) 396.

[20] L. F. Perondi, L. C. M. Miranda, J. Appl. Phys. 62
(1987) 2955.

[21] J. Alexandre, F. Saboya, B. C. Marques, M. L. P. Ribeiro, C. Salles, M. G. da Silva, M. S. Sthel, L. T. Auler, H. Vargas, The Analyst 124 (1999) 1209.

[22] L. Mota, R. Toledo, F. A. L. Machado, J. N. F. Holanda, H. Vargas, R. T. Faria Jr., Appl. Clay Sci. 42, 1-2 (2008) 168-174.

[23] S. Bribiesca, R. Equihua, L. Villaseñor, J. Eur. Ceram. Soc. 19 (1999) 1979.

[24] D. P. Almond, P. M. Patel, Photothermal Science and Techniques, Chapman \& Hall, London, Inglaterra (1996).

[25] I. Hatta, Rev. Sci. Instr. 50 (1979) 292.

[26] A. M. Mansanares, A. C. Bento, H. Vargas, N. F. Leite, L. C. M. Miranda, Phys. Rev. B 42 (1990) 4477.

[27] R. Bachmann, F. J. Disalvo Jr., T. H. Geballe, R. L. Greene, R. E. Howard, C. N. King, H. C. Kirsch, K. N. Lee, R. E. Schwall, H. U. Thomas, R. B. Zubeck, Rev. Sci. Instr. 43 (1972) 205.

[28] J. L. Pichardo, E. Marín, J. J. Alvarado-Gil, J. G. Mendoza-Alvarez, A. Cruz-Orea, I. Delgadillo, G. TorresDelgado, H. Vargas, Appl. Phys. A: Mater. Sci. Processing 65 (1997) 69.

[29] J. A. Balderas-López, S. A. Tomás, H. Vargas, V. OlaldePortugal, R. Baquero, I. Delgadillo, J. M. Yáñez-Limón, J. J. Alvarado-Gil, J. T. Frías-Hernandez, L. Scheinvar, C. Falcony, M. D. Silva, L. C. M. Miranda, Forest Products J. 46 (1996) 84.

[30] G. Gutiérrez-Juárez, O. Zelaya-Angel, J. J. AlvaradoGil, H. Vargas, H. O. Pastore, J. S. Barone, M. HernandezVelez, L. Baños, J. Chem. Soc. - Faraday Trans. 92 (1996) 2651.

[31] S. S. Gonçalves, M. G. da Silva, M. S. Sthel, S. L. Cardoso, R. R. Sanchez, J. B. Rieumont, H. Vargas, Physica Status Solidi A 187 (2001) 289.

[32] G. W. Brindley, M. Nakahira, J. Am. Ceram. Soc. 42 (1959) 311.

[33] I. Delgadillo, A. Cruz-Orea, H. Vargas, A. Calderón, J. J. Alvarado-Gil, L. C. M. Miranda, Optical Eng. 36 (1997) 343.

[34] G. W. Brindley, M. Nakahira, J. Am. Ceram. Soc. 42 (1959) 314.

[35] J. L. Holm, Phys. Chem. Chem. Phys. 3 (2001) 1362.

[36] J. E. Comeforo, R. B. Fischer, W. F. Bradley, J. Am. Ceram. Soc. 31 (1948) 254.

[37] D. R. dos Santos, R. Toledo, R. R. T. Faria Jr., J. G. Carrió, M. G. da Silva, L. T. Auler, H. Vargas, Rev. Sci. Instr. 74 (2003) 1.

[38] G. W. Brindley, S. Udagawa, J. Am. Ceram. Soc. 43 (1960) 59.

[39] K. Okada, N. Otsuka, J. Am. Ceram. Soc. 69 (1986) 652.

[40] K. Okada, N. Otsuka, J. Ossaka, J. Am. Ceram. Soc. 69 (1986) C251.

[41] R. J. Bratton, G. W. Brindley, J. Am. Ceram. Soc. 45 (1962) 513.

[42] R. Zallen, The physics of amorphous solids, John Wiley \& Sons, New York, EUA (1983). 
[43] E. Marín, J. Marín Antuna, P. Díaz Arencibia, Eur. J. Phys. 23 (2002) 523.

[44] W. D. Callister Jr., Ciência e Engenharia de Materiais:
Uma introdução, $5^{\text {a }}$ Ed., Ed. LTC, Rio de Janeiro, RJ (2002).

(Rec. 25/09/2008, Ac. 18/09/2009) 